\title{
Monitoramento das desigualdades sociais em saúde: significados e potencialidades das fontes de informação
}

\author{
M onitoring inequalities in health: \\ meanings and strengths of information sources
}

\begin{abstract}
This paper presents a conceptual analysis of inequalities in healthcare associated with the discussion of dimensions, indicators and data sources that are crucial for understanding major trends within the Brazilian context. From this standpoint, the text emphasizes the importance of in-depth discussions of social inequalities, identifying changes in healthcare conditions using the concept of differential vulnerability and the parameters that should be considered in the quest for strategic information required to draw up, implement, monitor and assess healthcare policies in this area.
\end{abstract}

Key words Social inequalities, Equity, Disparities in health, Information on health
Resumo 0 artigo busca desenvolver a reflexão conceitual sobre desigualdades sociais e iniqüidades em saúdearticulada à discussão dedimensões de análise, indicadores e fontes de dados a serem privilegiadas na caracterização de perfis e tendências no Brasil. N esta direção, o texto enfatiza a necessi dade de aprofundar o debate acerca das desigualdades sociais, identificando as variações na situação de saúde a partir do conceito de vulnerabilidade diferencial e os parâmetros a serem considerados na busca de informação estratégica para a formulação, implementação, monitoramento e avaliação de políticas públicas na área.

Palavras-chave Desigualdade social, Eqüidade, I niqüidades em saúde, I nformação em saúde 


\section{Introdução}

A pósa década de 1970, diferentes países do mundo ampliaram o debate sobre as desigualdades em saúdee, conseqüentemente, sobre o processo de conformação de práticas, ações eintervenções públicas socialmente mais efetivas na área. $\mathrm{A}$ publicação do Informe Lalonde pelo governo canadense em 1974, a estratégia "Saúde para Todos no Ano 2000" definida no âmbito da Assembléia M undial deSaúdeem 1977 ea realização da Conferência de Alma Ata em 1978 pela OM S representam importantes marcos nesta trajetória. Diferentes iniciativas de prevenção de doenças e promoção da saúde buscando ultrapassar o indivíduo e as famílias e abrangendo também 0 ambiente físico, sociocultural e político foram implementadas vis-à-vis a incorporação de novos referenciais teóricos. $\mathrm{Na}$ verdade, embora 0 estudo das desigualdades em saúdenão seja novo, as profundas mudanças institucionais e sociais decorrentes dos processos de globalização, da crise da condição salarial e da erosão da cidadania nas últimas décadas repercutiram nos perfis de morbidade e mortalidade da população e evidenciaram os limites do cuidado à saúde e das práticas assistenciais. Ao mesmo tempo, a necessidade de identificação mais precisa das variações nas situações de saúde e reorganização das políticas públicas a partir do estudo dos deslocamentos erupturas no perfil mais amplo de bemestar impôs esforços sistemáticos de investigação com o objetivo de melhorar a qualidade das fontes de informação.

Em grande parte, as dificuldades presentes na tarefa de mapear e selecionar as fontes de informação nos estudos sobre desigualdades sociais em saúde está ligada à própria natureza, dinâmica e complexidade do problema. Embora a afirmação de que o perfil epidemiológico das doenças eóbitos correlaciona-sefortemente com as condições socioeconômicas da população seja recorrente, a construção de indicadores e variáveis consistentes e, portanto, a busca de um conteúdo informacional aprimorado e capaz de incorporar as múltiplas dimensões das iniqüidades em saúde em diferentes contextos sociais representa um desafio. Como aponta Starfield", "a saúde está diretamente associada à vantagem social em termos absolutos. Quanto mais recursos sociais de indivíduos e comunidades, maior a probabilidade de uma saúde melhor". No entanto, é necessário discutir sobre que bases informativas devem ser construídos os parâmetros para a definição das condições sociais e de saúde dos diferentes grupos sociais. Para Januz$\mathrm{Zi}^{2}$, o indicador social é o "elo de ligação entre os model os explicativos da teoria social e a evidência empírica dos fenômenos sociais observados" e, portanto, estão envolvidos numa teia complexa designificadose dilemas metodológicos. Apesar das dificuldades, porém, é importante revigorar permanentemente 0 objetivo de garantir informação qualificada para o monitoramento de políticas e programas sociais voltados à equidade e ao bem-estar. Assim, este trabalho busca contribuir para o debate acerca das desigualdades sociais em saúde e das bases informacionais potencialmente capazes de subsidiar o processo de tomada de decisão e mudança institucional no âmbito das políticas na área. Frente ao crescenteinteressepelo tema eexistência de vasta literatura voltada à sistematização de conceitos e construção de ferramentas metodológicas capazes de refinar a pesquisa na área, a perspectiva aqui não é a de esgotar as múltiplas alternativas de análise mas, ao contrário, estimular a reflexão e novos aprendizados.

\section{Desigualdades sociais e iniqüidades em saúde}

Como apontam Marmot \& Wilkinson ${ }^{3}$, no sé culo XX, na maioria das sociedades democráticas e industrializadas, a expansão de sistemas de proteção social e de estratégias voltadas à meIhoria da qualidade de vida e saúde da população resultaram no aumento da expectativa de vida e na queda de indicadores de mortalidade e morbidade.

Neste cenário, o próprio tema das desigualdades em saúde perdeu centralidade na agenda públicae, apesar da persistência de limites eoportunidades diferenciadas no que se refere às condições de saúde dos indivíduos e grupos sociais somente nos final dos anos 70 e início dos anos 80 , o debate em torno do problema foi revigorado. Para os autores, a divulgação em 1982 do "Black Report"4 - relatório final do Research Working Group on Inequalities in H ealth organizado pelo governo britânico, representa uma importante inflexão na análise das relações entre saúdee condições sociais na I nglaterra. 0 estudo revisou as taxas de mortalidade segundo as diferentes classes socioeconômicas, a partir de $1911 \mathrm{e}$ nas décadas seguintes, e a análise dos dados revelou que, apesar das profundas modificações nas causas de morte, a tendência dos grupos sociais desfavorecidos apresentarem maiores taxas 
de mortalidade e menor esperança de vida ao nascer foi mantida ao longo do tempo.

0 relatório também promoveu um diálogo intenso sobre os problemas metodológicos da mensuração das desigualdades sociais em saúde. U ma das principais críticas direcionadas ao desenho da investigação foi a comparação das condições de saúde entre os grupos extremos dos perfis de distribuição de renda e estratificação social. O u seja, ao caracterizar o padrão das iniqüidades em saúde através do uso da média entre os mais e menos privilegiados socialmente, a pesquisa teria desconsiderado as desigual dades presentes entre os grupos. Ao mesmo tempo, a diminuição do número de profissionais com menor qualificação e renda e, inversamente, o aumento do contingente de trabalhadores mais qualificados entre 1930 e 1970 na Inglaterra teve importante influência na determinação das taxas de mortalidade no período. No entanto, apesar das possíveis limitações, o Black Report representou um importante esforço na retomada da problemática das desigualdades sociais em saúde nos países desenvolvidos. Com efeito, à luz das diferenças verificadas entre os perfis de morbidadee mortalidade dos indivíduos segundo sua posição social, a necessidade deestratégias eações públicas não focalizadas apenas no "setor saúde' ganhou mais força e consistência.

$\mathrm{N}$ a verdade, o foco no tema das desigualdades em saúde em um contexto social e político transformado impôs, no âmbito do processo de investigação epesquisa, a revisão de conceitosea articulação interdisciplinar. Assim, uma dasfundamentações mais relevantes introduzida no debate é oriunda do pensamento de Rawls ${ }^{5}$ sobre os conflitos e disputas políticas em torno dos princípios de justiça a serem pautados na distribuição de bens e ben efícios sociais. Para Rawls, a estrutura social comporta várias possibilidades de inserção e os indivíduos nessas diferentes posições ou lugares sociais têm distintas expectativas de vida e bem-estar. Algumas posições geram desigualdades nas chances iniciais dos indivíduos e, neste cenário, o autor propõe a justiça como eqüidade, combinando dois princípios: 0 da justiça formal, da igualdade de todos perante a lei e da justiça substantiva ou "real", enfatizando que as desigualdades socioeconômicas deverão ser regulamentadas para que resultem em benefícios compensatórios para todos e, especialmente, para os menos favorecidos. Portanto, para Rawls, a justiça como eqüidade implica defender o tratamento desigual para os desiguais.

No campo da saúde, a perspectiva da "discri- minação positiva" estreitamente vinculada ao debate sobre justiça social levou à tentativa de caracterização das iniqüidades ou desigualdades injustas definidas enquanto problemas que tendem a acompanhar as distâncias sociais existentes entre os indivíduos. N esta tarefa, M argareth Whitehead ${ }^{6}$ cunhou a definição de iniqüidades em saúde como" diferenças desnecessárias e evitáveis as quais são ao mesmo tempo consideradas injustas e indesejáveis". Ou seja, as iniqüidades em relação ao perfil de saúde são as desigualdades que podem ser contornadas e que impedem os indivíduos de alcançar plenamente seu potencial de bem-estar e de realização de "liberdades substantivas".

Segundo Braverman ${ }^{7}$, a definição de iniqüidade em saúde "concisa e acessível" proposta por Whitehead trouxe importantes contribuições para a investigação na área. Várias pesquisas foram desenvolvidas em diferentes conjunturas sociais, demonstrando um investimento técnico-científico abrangente e promovendo novos aprendizados. Em 1995, a Organização M undial deSaúde organizou um encontro com representantes de vários países do mundo em Genebra sobre Eqüidade e Atenção à Saúde. Um importante desdobramento do debate ocorrido neste fórum foi a perspectiva de construção de indicadores para o monitoramento da eqüidade em saúde em países ricos e pobres.

Alguns anos mais tarde, o estudo "Solids Facts"8, publicado pela Oficina Regional para a Europa da OM S em 2003 sobre as evidências que iluminam as ligações entre as circunstâncias sociais ea saúde dos indivíduos, reconheceu que os ricos vivem mais e ficam menos doentes que os pobres tanto nos países desenvolvidos como nos países em desenvolvimento. Segundo o estudo, as desvantagens no que se refere à saúde das pessoas, de acordo com a pesquisa, embora apresentem múltiplas formas e especificidades tendem a se concentrar entre grupos socialmente vulneráveis e ter efeitos cumulativos. Assim, a bai xa escolaridade influencia as decisões familiares sobre alimentação, cuidados com o corpo e prevenção de doenças e tende, desta forma, a ter profundo impacto nas condições de saúde. A experiência ocupacional que exige intenso esforço físico e emocional eque freqüentemente associaseà redução de expectativas eaumento do estresse está intimamente relacionada ao surgimento de doenças cardiovasculares. Por outro lado, 0 desemprego enquanto situação que compromete 0 acesso à renda, bens e serviços e, também, fragiliza a auto-estima, a interação social e a 
identidade pessoal, tem profundas repercussões nas condições de saúde. 0 documento assinala ainda as repercussões do padrão de coesão social, definida com basena existência de circuitos de reciprocidade e confiança mútua, na redução do estresse, da depressão e das doenças crônico-degenerativas.

N este esforço recente voltado à reflexão sobre as possíveis mediações entre situação de saúde e desigualdades sociais, vários autores têm estudado os processos em que são combinados diferentes aspectos da privação objetiva e subjetiva na trajetória cotidiana dos indivíduos. Como apontam Wagstaff, Paci \& Joshi ${ }^{9}$, certos momentos da vida que implicam "transições críticas" como a infância, a adolescência, a passagem do nível fundamental para o secundário na escola, 0 primeiro emprego, a formação de uma nova família, a mudança de emprego ea aposentadoria ganham, assim, especial interesse na medida em que evidenciam, freqüentementedemaneiradrástica, oportunidades diferenciadas no que se refere à mobilidade material, ao acesso ao capital cultural e simbólico e, portanto, à capacidade de superar rupturas e mudanças. A compreensão do "gradiente social" da saúde implicaria, desta forma, compreender processose circuitos de destituição em geral interdependentes.

\section{Vulnerabilidade e exposição diferencial: as dimensões das desigualdades em saúde}

O reconhecimento da existência de uma "cadeia de eventos" que articula diferentes mecanismos de integração, inclusão e exclusão sociais reorienta a abordagem sobre as causas e dimensões das desigualdades em saúde. Uma das perspectivas mais importantes deste processo de reorientação analítica é a ênfase em "ciclos de vida" e trajetórias singulares dos grupos sociais, a fim de caracterizar a vulnerabilidade e a exposição diferencial aos agravos à saúde. 0 estudo dos chamados "fatores de risco individuais" dá lugar, assim, a enfoques mais amplos que privilegiam o perfil de acesso a políticas públicas, padrões de consumo e redes de interação social

Entender os diferentes perfis de doença esuas mediações sociais ultrapassa, portanto, o estudo de variações biológicas e incorpora o foco na dimensão social das vulnerabilidades e nos mecanismos complexos que sustentam a relação entre a dinâmica das desigualdades e as condições de saúde. Assim, os conceitos de exposição evulnerabilidadesocial envolvem a compreensão deque "o impacto sanitário de uma exposição concreta dependerá da existência ou não de outras causas ou fatores de risco associados. Como as pessoas inseridas em posições sociais menos privilegiadas são expostas a muitos fatores de risco diferentes ecomo estes fatores podem interagir entre si, a vulnerabilidade ao efeito de uma causa específica tendea ser maior nestes grupos do que entre os membros das setores sociais mais privilegiados" 10 .

Observando mais de perto como operam os principais mecanismos de intermediação entre condições de saúde, comportamentos individuais e desigualdades sociais, Robert Evan ${ }^{11} \mathrm{de}$ monstrou a baixa capacidade explicativa dos fatores de risco individuais na conformação do perfil demortal idadeentrediferentes grupos profissionais. A partir do resultado do estudo de M armot et al. ${ }^{12}$ e utilizando o exemplo do tabagismo, o autor concluiu que os grupos sociais mais privilegiados na hierarquia profissional raramente apresentam o hábito de fumar. Além disso, as causas de óbito entre os poucos tabagistas presentes nestes grupos, em geral, não se correlacionam com o "fator derisco". Para Evans, o fumo bem como os altos níveis de colesterol sanguíneo e pressão arterial parecem expressar, ao invés de causas de morbidade e mortalidade, elos ou "terrenos compartilhados" entre o espaço das diferenças socioeconômicas e os indicadores de saúde.

Torna-se importante, assim, evitar analisar de maneira isolada os efeitos dos aspectos sociais e econômicos na saúde. Apesar de existir um certo consenso de que a pobreza e piores condições de saúde se articulam numa "causalidade bidirecional", é importante reconhecer que a pobreza e a desigual dade social têm múltiplas faces e dimensões. Caracterizar as situações de vulnerabilidade social e sua relação com a saúde envolve, portanto, lidar com conceitose noções que ultrapassam fronteiras disciplinares.

\section{Medindo as desigualdades em saúde: dilemas edesafios}

A partir das considerações anteriores, surge como um dos principais desafios para a mensuração das desigualdades sociais em saúde a seleção de indicadores sociais relevantes. Ao mesmo tempo, é necessário discutir permanentemente 0 alcance dos indicadores selecionados a partir de sua repercussão na saúde e, também, de sua capacidade de dar visibilidade a dinâmicas de interação. A renda, por exemplo, pode ter em im- 
pacto importante no risco de adoecer e morrer precocemente, mas pode explicar pouco certos perfis epidemiológicos se não for associada a outras variáveis. Como apontam Wagstaff, Paci \& Joshi ${ }^{13}$, apesar da renda ser importante no nível individual para explicar as condições de saúde, quando se estende o foco para segmentos maiores da população, é importante levar em consideração outros fatores ligados às relações sociais. Para os autores, o efeito da renda nas condições de saúde pode ser neutralizado onde existem maiores investimentos em infra-estrutura e equipamentos coletivos. Ainda que haja uma articulação entre renda per capita e condições de saúde, alguns estudos mostram que na medida que se avança nas classes de renda, o efeito desta variável tende a diminuir. Ou seja, o aumento da renda parece ter mais impacto na saúde dos mais pobres.

Por outro lado, a desigualdade derenda entre os grupos sociais - a chamada pobreza relativa refletida nos indicadores de concentração derenda e também as diferentes formas de privação para além da privação material e absoluta têm uma associação mais positiva com as diferenças na esperança de vida ao nascer, mortalidade infantil e nas diferentes faixas etárias e morbidade. Isto porque as sociedades que apresentam maiores índices de desigualdade social também tendem a apresentar menores investimentos em capital humano esocial. Wilkinson ${ }^{14}$ analisou o caso de países como a Inglaterra e a I tália em diferentes períodos históricos, buscando uma associação entre desigualdade de renda, capital social, solidariedade, vida associativa, esperança de vida ao nascer etaxas de mortalidade. De acordo com os resultados da pesquisa, em 1960 na Itália, o recrudescimento das desigual dades de renda entre os grupos sociais além de ameaçar e romper a coesão social também promoveu o aumento da mortalidade por doenças coronarianas. Ainda a partir da investigação, os autores apresentaram evidências importantes dequenal nglaterra após a II Guerra M undial, uma trajetória socioeconômica inversa e o fortalecimento da idéia de pertencimento à nação influenciaram o aumento da expectativa de vida, reforçando a tese de quealém da privação material absoluta, heterogeneidades intensas nos níveis de bem-estar, isolamento, falta de suporte emocional e riscos acentuados de fratura social tendem a ocasionar maiores problemas de saúde na população. N esta direção, Lochner e Kawachi ${ }^{15}$ mostram que as características dos "lugares" e os níveis de capital social existentes nos diferentes contextos locais influenci- am a saúde e impactam as taxas de mortalidade por causas violentas, doenças cardiovasculares e câncer.

Também éimportante distinguir pobreza real e socialmente percebida. M uitas vezes, a distribuição desigual da riqueza pode ser agudizada sem que a percepção da sociedade seja modificada e vice-versa ${ }^{16}$. A pobreza também pode estar associada à falta de expectativa ou aos constrangimentos ligados à dificuldade de manter vínculos elaços sociais. Como analisa Peter Townsend ${ }^{17}$ a humilhação das famílias por não conseguirem contribuir na Igreja a qual fazem parte, por não participar de alguma festividade ou outras atividades de lazer pode fragilizar a convivência e criar isolamento social. Essas questões, para o autor, fazem parte da definição de pobreza assim como a fome ou a falta de moradia. N esta leitura"compreensiva" da pobreza, processos de construção de identidade que resultam em estigma devem, também, ser objeto de atenção na análise do perfil das iniqüidades em saúde.

No entanto, persistem importantes controvérsias sobre como agregar e correlacionar as informações sobre situação social e condições de saúde. Com isso, é possível perceber perspectivas distintas e muitas vezes conflitantes sobre 0 monitoramento das iniqüidades em saúde e dos rumos e estratégias capazes de garantir a construção de bases de dados e informação qualificada na área. De um lado, prioriza-se o estudo das diferenças no perfil sanitário entre indivíduos quase a despeito de suas condições sociais e, de outro lado, as comparações entre distintas experiências de saúde e doença são balizadas pela definição prévia dos grupos que, sistematicamente, acumulam déficits e desvantagens no que se refere ao acesso a bens públicos e oportunidades sociais. N estecaso, a eqüidadeé entendida como a ausência de diferenças nas condições de saúde entre os segmentos mais e menos privilegiados socialmente.

Sem dúvida, algumas tentativas de aproximação e intermediação entre as diferentes abordagens têm possibilitado interpretações menos polarizadas sobre os parâmetros que devem orientar a mensuração das desigual dades sociais em saúde. Examinando as diferenças nos resultados em saúde que tendem a favorecer grupos em desvantagem social, por exemplo, muitos estudos têm trazido implicações cruciais para a investigação na área. Um dos avanços mais significativos neste processo de complexificação do tema da mensuração das desigualdades em saúde é o cuidado com a comparação entre indicadores de 
saúde de grupos menos favorecidos com a média da população em geral, na medida em que tende a obscurecer as diferenças relativas entre os grupos sociais. Também, a revisão de pressupostos e metodologias tem iluminado a necessidade de estudar e mensurar as condições que produzem diferenças no processo de reabilitação e superação dos problemas de saúde. As famílias que possuem maiores chances de mobilizar recursos para o apoio e proteção de seus membros em caso de doença têm experiências bastante distintas ligadas a seqüelas e incapacidades físicas e emocionais em comparação às famílias desprotegidas e isoladas socialmente ${ }^{18}$. A busca de indicadores sensíveis a esta realidade diferenciada torna-se, portanto, prioritária.

No Brasil, cresce o debate sobre os perfis epidemiológicos nos diferentes grupos sociaise, também, sobre as profundas diferenças regionais relativas aos serviços de saúde no que se refere à capacidadeinstalada, ao financiamento das ações, ao acesso eao padrão deutilização. A reflexão em torno das possibilidades de consumir serviços de saúdenos diferentes níveis de complexidadeentre os indivíduos que teriam as "mesmas necessidades de saúde" ou a eqüidade horizontal tem sido combinadaà questão do tratamento desigual para indivíduos com necessidades distintas, definido como equidade vertical ${ }^{19}$.

A través das bases de dados com periodicidade anual e abrangência nacional como os censos demográficos e o Datasus, por exemplo, é possível mapear principalmenteas desigual dades geográficas, de gênero e faixa etária e suas relações com o perfil de saúde e doença. Segundo os dados da Pesquisa Nacional por Amostras de Domicílios ( PNAD ) 200320, ainda que 0 acesso à saúde entre os mais pobres tenha aumentado no país entre 1998 e 2003, prevalece a desigual dade entre os diferentes estados e municípios. Nas áreas rurais, a proporção de pessoas que realizaram pelo menos uma consulta médica no ano anterior à pesquisa permaneceu menor do que nas regiões urbanas. No que se refere à cobertura de mamografia, por exemplo, embora a média no país tenha alcançado $41,2 \%$, nos estados deTocantins, M aranhão eParaíba, cerca de 74 \% das mulheres na faixa etária acima de 50 anos nunca realizaram o exame.

Pesqui sas específicas como a Pesquisa de $0 \mathrm{r}$ çamento Familiar (POF) e a Pesquisa Nacional de Saúde e N utrição (PNSN), apesar de não serem regulares, também têm fornecido impor- tantes informações e dados para a análise das correlações entre condições sociais e saúde no Brasil. Um exemplo oportuno de pesquisa voltada ao aperfeiçoamento das informações sobre desigualdades em saúde no Brasil foi o estudo exploratório Epidemiologia das Desigualdades em Saúde no Brasil- OPAS/M S'21. Analisando dados nacionais de mortalidade, natalidade, morbidade e também de oferta e cobertura dos serviços de saúde, o estudo trouxe elementos importantes para a compreensão do padrão assimétrico dos indicadores de saúde nas diferentes regiões do país. No entanto, 0 trabalho reconhece a falta de uma "cultura informacional" capaz de produzir o registro acurado de dados regionalizados sobre questões substantivas para a análise das desigualdades em saúde, como situação ocupacional, étnica e educacional. Ainda que o desenvolvimento recentedenovas tecnologias para o processamento e divulgação de dados relevantes seja um avanço inegável, o planejamento de ações voltadas ao enfrentamento das desigualdades em saúde no país ainda ébastante dificultado pela fragilidade das fontes de informação.

Para N unes et al. ${ }^{22}$, o preenchimento incorreto e insuficiente da declaração de óbito e dos re gistros hospitalares, na maioria das vezes, inviabiliza a produção de informação integrada sobre o panorama das iniqüidades em saúde. Os dados de renda e situação ocupacional na maioria das vezes não são levantados, obstaculizando 0 estudo mais rigoroso do impacto destas variáveis sociais no perfil sanitário da população. De outra parte, a falta de investimentos sistemáticos em pesquisas longitudinais sobre a dinâmica de interação entre condições sociais e saúd de fragiliza o alcance de parâmetros mais consistentes para a ação pública voltada à garantia de eqüidade.

Como ressalta Braverman ${ }^{23}$, a pesquisa e a investigação pública certamente não são suficientes para a redução das desigualdades em saúde, mas sem monitorar como as disparidades entre os grupos mais e menos privilegiados socialmente são transformadas ao longo do tempo e sua relação com as políticas públicas, torna-se difícil garantir accountability em relação aos diferentes efeitos das ações entre os segmentos vulneráveis. Neste sentido, o fortalecimento e manejo qualificado da informação sobre desigualdades em saúde podem contribuir para a construção de cenários mais eqüitativos no campo das políticas de saúde. 


\section{Considerações finais}

Para Amartya Sen, "a necessidade de admitir a incompletudena avaliação da desigualdade éinescapável"24. N estesentido, limites, ambigüidadese lacunas no estudo das iniqüidades em saúde devem ser tematizados e integrados ao esforço analítico-descritivo em oposição à busca de modelos explicativossimplistas elineares. 0 entendimento da magnitude das diferenças nas condições de saúde dos grupos sociais representa, portanto, tarefa em permanente reformulação e reavaliação. Como aponta a literatura na área, as cone- xões e graus de influência dos diferentes aspectos da realidadesocial eeconômica na experiênciaindividual ecoletiva de saúde, na maioria das vezes, não podem ser percebidos imediatamente. Situar o problema do monitoramento das desigualdades em saúde associadas ao perfil das vulnerabilidades sociais é compreen der a necessidade de fazer dial ogar diferentes disciplinas efiliaçõesteórico-metodológicas. Somente a partir desta perspectivainterdisciplinar épossível construir novas e significativas bases informacionais para o desenho e implementação de políticas de saúde capazes de dar resposta ao desafio da eqüidade.

\section{Referências}

1. Starfield B. Atenção primária. Brasília: Unesco/M S; 2002.

2. Januzzi PM. Indicadores sociais no Brasil - conceitos, fontes de dados e aplicações. São Paulo: Editora Alínea; 2004.

3. Marmot $M$, Wilkinson R. Social determinants of health. New York: Oxford University Press; 1999.

4. Townsend P, Davidson N. The Black Report. London: Pelican Books; 1982.

5. Rawls J. U ma teoria da justiça. São Paulo: Martins Fontes; 1997.

6. Whitehead $M$. The health divide. In: Townsend $P$ Whitehead M, Davidson N, eds. Inequalities in $\mathrm{He}$ alth: The Black Report and the Health Divide. London: Penguin; 1992. p.214-450.

7. Braverman, P. Health Disparities and Health Equity: Concepts and Measurement. Annu Rev Public Health 2006; 27:167-194.

8. WHO Europe. Social Determinants of $\mathrm{Health}$ : The Solid Facts. Copenhagen: WH O; 2003.

9. Wagstaff $A$, Paci $P$, Joshi $H$. Inequalities in $\mathrm{H}$ ealth Who you are? Where you live? Who your parents were? World Bank Policy Research, Working Paper n.2713; 2001.

10. Diderichsen F, Evans T, Whitehead M. Bases sociales de las disparidades en salud. In: Evans T, organizador. Desafio a la falta de equidad en la salud. Washington: OPS/Fundação Rockefeller; 2002. p.13-26

11. Evans RG, Bazer ML, M armor TR, eds. Why are some people healthy and others not? The Determinants of Health of Populations. New York: Aldine de Gruyter; 1994.

12. M armot M G, Adelstein AM, Robinson N, Rose GA. Changing social-class distribution of heart disease. Br M ed J 1978, 2 (6145): 1109-12.

13. Wagstaff $A$, Paci $P$, Joshi $H$. Inequalities in $H$ ealth: Who you are? Where you live? Who your parents were? World Bank Policy Research, Working Paper n.2713; 2001.
14. Wilkinson RG. Unhealthy Societies:Affliction of Inequality. London: Routledge; 1996.

15. Lochner KA, Kawachi I, Brennan RT, Buka SL. Social capital and neighborhood mortality rates in Chicago. Social Science \& M edicine 2003; 56:1797-1805.

16. Rocha S. Governabilidade e pobreza: o desafio dos números. In: Valladares $L$, Coelho $M$, organizadoras. Governabilidade e pobreza no Brasil. Rio de Janeiro: Civilização Brasileira; 1995.

17. Townsend $P$. The International Analisys of Poverty. New York: Harvester Wheatsheaf; 1993. p.27-39.

18. Braverman P. Health Disparities and Health Equity: Concepts and M easurement. Annu. Rev. Public Health 2006; 27:167-194.

19. Travassos $C$, et al. Utilização dos serviços de saúde no Brasil: gênero, características familiares e condição social. Revista Panamericana de Salud Pública 2002; 11:365-373.

20. IBGE - Pesquisa Nacional de Amostra de Domicílios (PNAD) - Síntese de Indicadores 2003.

21. OPAS/M S. Epidemiologia das desigualdades em saúde no Brasil: um estudo exploratório. Brasília: OPAS/ MS; 2002

22. Nunes $A$, et al. M edindo desigualdades em saúde no Brasil. Brasília: OM S/OPAS/IPEA; 2001.

23. Braverman P. Health Disparities and Health Equity: Concepts and M easurement. Annu. Rev. Public Health 2006; 27:167-194.

24. Amartya S. Desigualdade reexaminada. Rio de Janeiro: Editora Record; 2001. p.43-60.

Artigo apresentado em 29/06/2006

Aprovado em 26/09/2006

Versão final apresentada em 24/11/2006 